\title{
Resistance to compression of weakened roots subjected to different root reconstruction protocols
}

\author{
Lucas Villaça ZOGHEIB ${ }^{1}$, Guilherme de Siqueira Ferreira Anzaloni SAAVEDRA², Paula Elaine CARDOSO ${ }^{3}$, Márcia $^{2}$ \\ Carneiro VALERA ${ }^{4}$, Maria Amélia Máximo de ARAÚJO ${ }^{4}$
}

\begin{abstract}
1- DDS, MSc, PhD, Professor, School of Dentistry, Sagrado Coração University, Bauru, SP, Brazil.
2- DDS, MSc, PhD, Associate Professor, Department of Restorative Dentistry, School of Dentistry of São José dos Campos, Univ. Estadual Paulista - UNESP, São José dos Campos, SP, Brazil.

3- DDS, MSc, PhD, Department of Restorative Dentistry School of Dentistry of São José dos Campos, Univ. Estadual Paulista - UNESP, São José dos Campos, SP, Brazil.

4- DDS, MSc, PhD, Associate Professor, Department of Restorative Dentistry School of Dentistry of São José dos Campos, Univ. Estadual Paulista - UNESP, São José dos Campos, SP, Brazil.
\end{abstract}

Corresponding address: Lucas Villaça Zogheib - Universidade de Sagrado Coração/Faculdade de Odontologia - Rua Irmã Arminda, 10050 - Bauru, SP 17011-160 - Brasil - Phone: (14) 81530479 - e-mail: lucaszogheib@yahoo.com.br

Received: June 10, 2009 - Modification: March 17, 2010 - Accepted: 27 April, 2011

\section{ABSTRACT}

\begin{abstract}
bjective: This study evaluated, in vitro, the fracture resistance of human non-vital teeth restored with different reconstruction protocols. Material and methods: Forty human anterior roots of similar shape and dimensions were assigned to four groups $(n=10)$, according to the root reconstruction protocol: Group I (control): non-weakened roots with glass fiber post; Group II: roots with composite resin by incremental technique and glass fiber post; Group III: roots with accessory glass fiber posts and glass fiber post; and Group IV: roots with anatomic glass fiber post technique. Following post cementation and core reconstruction, the roots were embedded in chemically activated acrylic resin and submitted to fracture resistance testing, with a compressive load at an angle of $45^{\circ}$ in relation to the long axis of the root at a speed of $0.5 \mathrm{~mm} / \mathrm{min}$ until fracture. All data were statistically analyzed with bilateral Dunnett's test $(\alpha=0.05)$. Results: Group I presented higher mean values of fracture resistance when compared with the three experimental groups, which, in turn, presented similar resistance to fracture among each other. None of the techniques of root reconstruction with intraradicular posts improved root strength, and the incremental technique was suggested as being the most recommendable, since the type of fracture that occurred allowed the remaining dental structure to be repaired. Conclusion: The results of this in vitro study suggest that the healthy remaining radicular dentin is more important to increase fracture resistance than the root reconstruction protocol.
\end{abstract}

Key words: Nonvital tooth. Tooth root. Permanent dental restoration. Post and core technique. Compressive strength. Tooth fractures.

\section{INTRODUCTION}

The success achieved with esthetic restorative treatments has resulted in an increasing demand by patients for these treatments, especially for anterior teeth ${ }^{17,22}$. Consequently, there has been a significant increase in the use of ceramic crowns, as well as intraradicular posts and core materials with satisfactory mechanical and esthetic properties ${ }^{4,9}$. Prefabricated intraradicular posts are preferred because they are more practical, economic, and in some situations, less invasive than custom-made cast metal cores $^{18,21,23,25}$.
Intraradicular posts are necessary for restoring the crowns of teeth compromised by endodontic treatment, with widened canals. Several authors have affirmed that the need to use intraradicular posts is determined by two main factors: the quantity of remaining dentin for retaining the crown, and the internal nature of the root structure ${ }^{4,7,24}$. Factors such as, caries and trauma may create a widened root canal. In cases of dental trauma in young patients, these frequently interrupt apical closing and complete root development, leading to the formation of an widened root canal that remains like that ${ }^{10,11}$. Widened canals are more susceptible to fracture because of the thin remaining walls, 
requiring restorative techniques that do not compromise the integrity of the remaining root structure ${ }^{11,30}$. A method that has been shown to substantially increase fracture resistance of endodontically treated teeth is the placement of a cast metal band around the coronal surface, leaving it above this margin. This procedure, known as the "ferrule effect", is recommended by many authors when the fracture resistance of endodontically treated teeth needs to be increased $5,12,14,19,24$. With the introduction of materials that bond to dentin, the possibility arose of reconstructing and consequently, rehabilitating root canals severely damaged by caries, trauma, congenital disorders, and internal reabsorption ${ }^{15}$.

The use of intraradicular posts, which can be bonded both to dentin and to the core, has been defended. They improve the distribution of stresses along the root and consequently, contribute to reinforcing the structure ${ }^{9-11,15,20,26,27}$. Researches involving intraradicular posts and cores are moving in the direction of developing systems that are strong, resistant to corrosion and biocompatible ${ }^{23}$. In this context, resin-reinforced intraradicular posts have been increasingly tested, especially those reinforced with glass fiber $1,2,6,13,16,29,30$.

The use of fiber posts in weakened and flared root canals has some limitations namely misfit, especially at the coronal level where the resin cement layer would be excessively thick, and bubbles are possible to form within it, predisposing to debonding failure ${ }^{21}$. Different techniques have been proposed to minimize this problem $5,6,28$. Grandini, et al. ${ }^{6}$ (2003) described a technique named anatomic post. In this technique, the fiber post is relined into the root canal replacing the resin cement by the composite resin, which has better mechanical and physical properties. The advantages of this technique are increase the internal thickness of resin on root walls and reduce the resin cement thickness ${ }^{6}$. On the other hand, there is the difficulty to polymerize the composite resin in more apical regions of the root canal. Solomon and Osman ${ }^{28}$ (2003) report a case where a wide flared root canal was rehabilitated with composite resin increments using a light-transmitting plastic post. Another technique cited by Clavijo, et al. ${ }^{5}$ (2009), combines a main glass fiber post with accessories fiber posts, which presents a small diameter.

This study compared three techniques of root reconstruction using composite resin and glass fiber posts. The null hypothesis is that there is no statistically significant difference in the fracture resistance and failure mode between roots reconstructed in the different ways.

\section{MATERIAL AND METHODS}

Forty roots of human anterior teeth of similar shape and dimensions were obtained from teeth extracted for periodontal or orthodontic reasons at the clinic of the School of Dentistry of São José dos Campos (UNESP), Discipline of Surgery. The study protocol was approved by the local Research Ethics Committee (031/07-PH/CEP). The inclusion criteria of the roots were: straight roots with mesiodistal dimensions between 5.0 and $5.5 \mathrm{~mm}$ and buccolingual dimensions between 7.0 and 8.0 $\mathrm{mm}$ (cross section). Roots with widened canals or apical dilacerations, fissures or surface defects were excluded. After selection, the roots were scaled with a periodontal curette (Duflex, Rio de Janeiro, RJ Brazil), sectioned to a standardized length of $13 \mathrm{~mm}$ with a diamond double-faced disk (KG Sorensen, Barueri, SP, Brazil) mounted in a lowspeed handpiece (Kavo, Joinville, SC, Brazil) cooled with air water spray and stored in distilled water until they were used (Figure $1 \mathrm{~A}$ ).

Root preparation and restorative procedures

All roots were initially prepared with a largo drill, $1 \mathrm{~mm}$ in diameter, number 1 from the glass fiber post kit (Reforpost Fiber Glass X-Ray, Angelus, Londrina, PR, Brazil) to a length of $9 \mathrm{~mm}$ (Figure $1 B$ ) and were randomly distributed into 4 groups $(n=10)$ in accordance with the root reconstruction protocol:

Group I - Control: the roots in this group were prepared to receive a glass fiber post, but did not receive internal reduction. After preparation, the root was restored with a glass fiber post.

Group II - Composite resin increments and glass fiber post: the roots in this group were prepared and weakened to simulate roots with wide canals and thin dentinal walls in the middle and cervical thirds using a tapered rounded end diamond bur (No. 2135, KG Sorensen, Barueri, SP, Brazil) with an 8 -mm-long active tip (Figure $1 \mathrm{C}$ ). With the aid of a thickness meter, constant measurements were made until dentinal walls with a thickness of $1 \mathrm{~mm}$ in the cervical third were obtained (Figure 1D). The weakened root was reconstructed with composite resin by the incremental technique and glass fiber post. To make the root reconstruction in this group, increments of hybrid composite resin (Filtek Z250, 3M/ESPE Dental Products, St. Paul, MN, USA) of a maximum of $2 \mathrm{~mm}$ in diameter, were inserted from the middle third until the root was filled. The lighttransmitting plastic post (Luminex, Dentatus, New York, NY, USA), corresponding to the root canal, was used as a reference for obtaining the post space after increments were light activated.

Group III - Main glass fiber post with accessories fiber posts: the roots in this group were prepared 

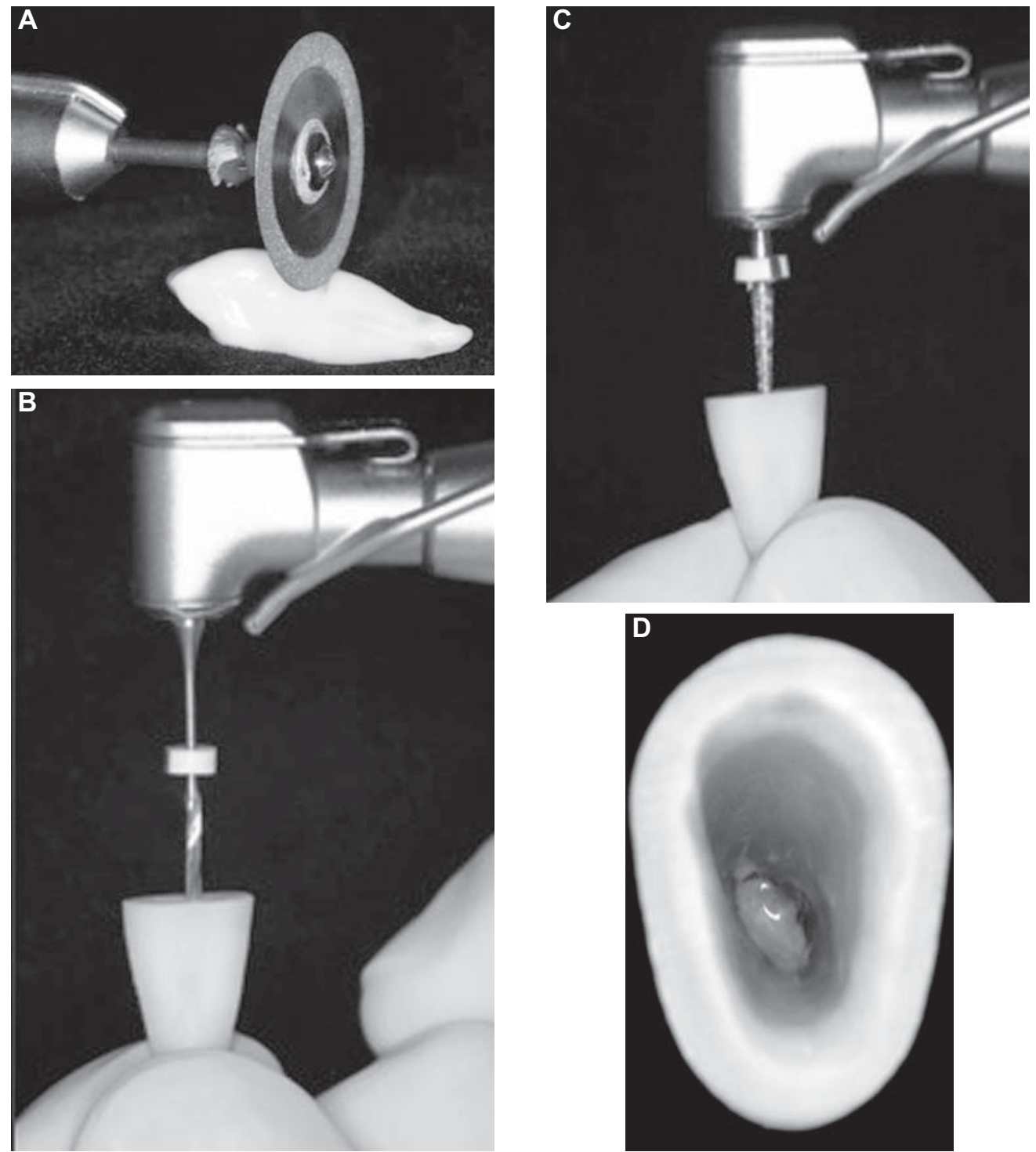

Figure 1- Root sectioning (A), initial intraradicular preparation with largo bur (B), final intraradicular preparation with a tapered rounded end diamond bur $(C)$, weakened root (D)

and weakened as described in group II. The weakened root was reconstructed by cementing the main glass fiber post and three accessories glass fiber posts (Reforpin, Angelus, Londrina, PR, Brazil).

Group IV - Anatomic post: the roots in this group were prepared and weakened as described in group II. The weakened root was reconstructed by the anatomic post technique (glass fiber post, surrounded by light activated resinous cement). Roots in this group, received a layer of liquid glycerin before the resinous cement was inserted, so that the polymerized post plus cement could be removed after initial light activation (20 s). Next, the anatomic post was light activated for a further 20 s, followed by cementation. Afterwards, the external portion of all the posts was sectioned with a diamond disk under water cooling, so that a $4 \mathrm{~mm}$ height remained outside the canal.

In all groups, the glass fiber posts (Reforpost Fiber Glass X-Ray, Angelus) were cleaned with
$96 \%$ alcohol, treated with a silane agent (Ceramic Primer-Silano RelyX, 3M/ESPE, St. Paul, MN, USA) and cemented with a dual cure resin-based cement (Rely $X, 3 M / E S P E$ ) using a single-bottle adhesive (Single Bond, 3M/ESPE) in accordance with the manufacturers' instructions for root preparation.

\section{Core fabrication}

To standardize the fabrication of the coronal portion of the hybrid composite resin core (Filtek Z250, 3M/ESPE), silicon matrixes were made, having as reference a maxillary canine made of chemically activated acrylic resin, previously prepared for an all-ceramic crown. These silicon matrixes were filled with composite resin, placed on the posts and light activated for $40 \mathrm{~s}$. At the end, the matrix was removed, the excess of composite resin was eliminated and the 5-mm-high filling core was obtained. 


\section{Obtaining the specimens}

To simulate the periodontal ligament, the roots were covered with a uniform layer of $\mathrm{n}^{0} 7$ wax, 2 $\mathrm{mm}$ below the cervical margin and embedded in metal cylinders $(60 \mathrm{~mm}$ in diameter and $20 \mathrm{~mm}$ high) with chemically activated acrylic resin (Jet Clássico, Artigos Odontológicos Clássico Ltd., São Paulo, SP, Brazil). The set was immersed in water at $75^{\circ} \mathrm{C}$ for $1 \mathrm{~min}$ to remove the wax layer, leaving a space between the root and the acrylic resin. A polyether-based impression material (Impregum, 3M/ESPE) was prepared and applied around the
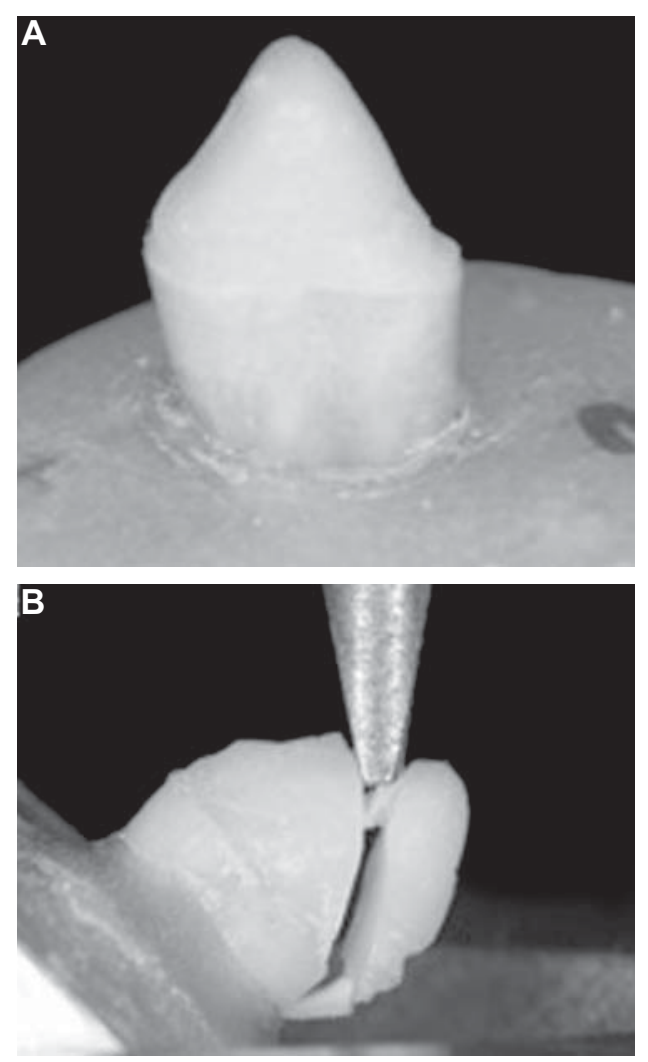

Figure 2- Specimens before $(A)$ and after $(B)$ mechanical testing root surface. The root was again put into the resin cylinder and after the material was polymerized, the excess was removed with a scalpel blade at the predetermined level of $2 \mathrm{~mm}$ (Figure 2A).

\section{Fracture-resistance testing}

The specimens were subjected to the fracture resistance test in a universal testing machine (EMIC DL 2000; EMIC, São José dos Pinhais, PR, Brazil). A compressive force was applied on the lingual surface ( $2 \mathrm{~mm}$ below the incisal edge) at an angulation of $45^{\circ}$ and speed of $0.5 \mathrm{~mm} / \mathrm{min}$ until fracture (Figure 2B).

The failure mode of each specimen was classified into: 1) repairable (displacement of the nucleus or root fracture above the root cervical level which would allow a new restoration) or 2) irreparable (fracture below the root cervical third, vertical or oblique fracture and horizontal facture in the middle and apical thirds which condemn the tooth to extraction).

\section{Statistical analysis}

The mean fracture resistance values recorded in $\mathrm{N}$ were analyzed statistically with the bilateral Dunnett's test at a significance level of $a=5 \%$.

\section{RESULTS}

Statistically significant difference $(p<0.05)$ was found between the experimental groups and the control group. The comparison of the 3 experimental groups among them showed no statistically significant difference $(p=0.5868)$. Figure 3 shows the mean fracture values obtained with the different root reconstruction protocols.

The quantitative distribution of the failure modes is presented in Figure 4 and Figure 5 shows the qualitative sample representation of the failures after the mechanical test.

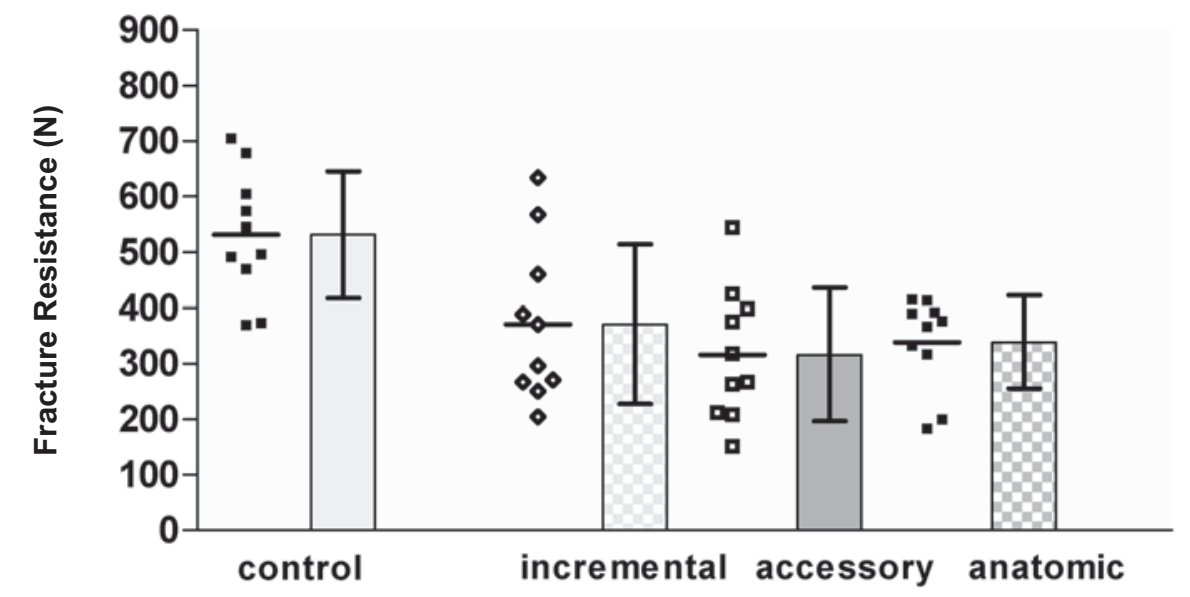

Figure 3- Points diagram around the mean and column graph (mean \pm standard deviation) of the fracture resistance values $(\mathrm{N})$ for the tested roots 


\begin{tabular}{|c|c|c|c|c|}
\hline Failure Mode & Group 1 & Group 2 & Group 3 & Group 4 \\
\hline Repairable & 10 & 8 & 6 & 5 \\
\hline Irreparable & 0 & 2 & 4 & 5 \\
\hline Total & 10 & 10 & 10 & 10 \\
\hline
\end{tabular}

Figure 4- Frequency of failure mode after mechanical test
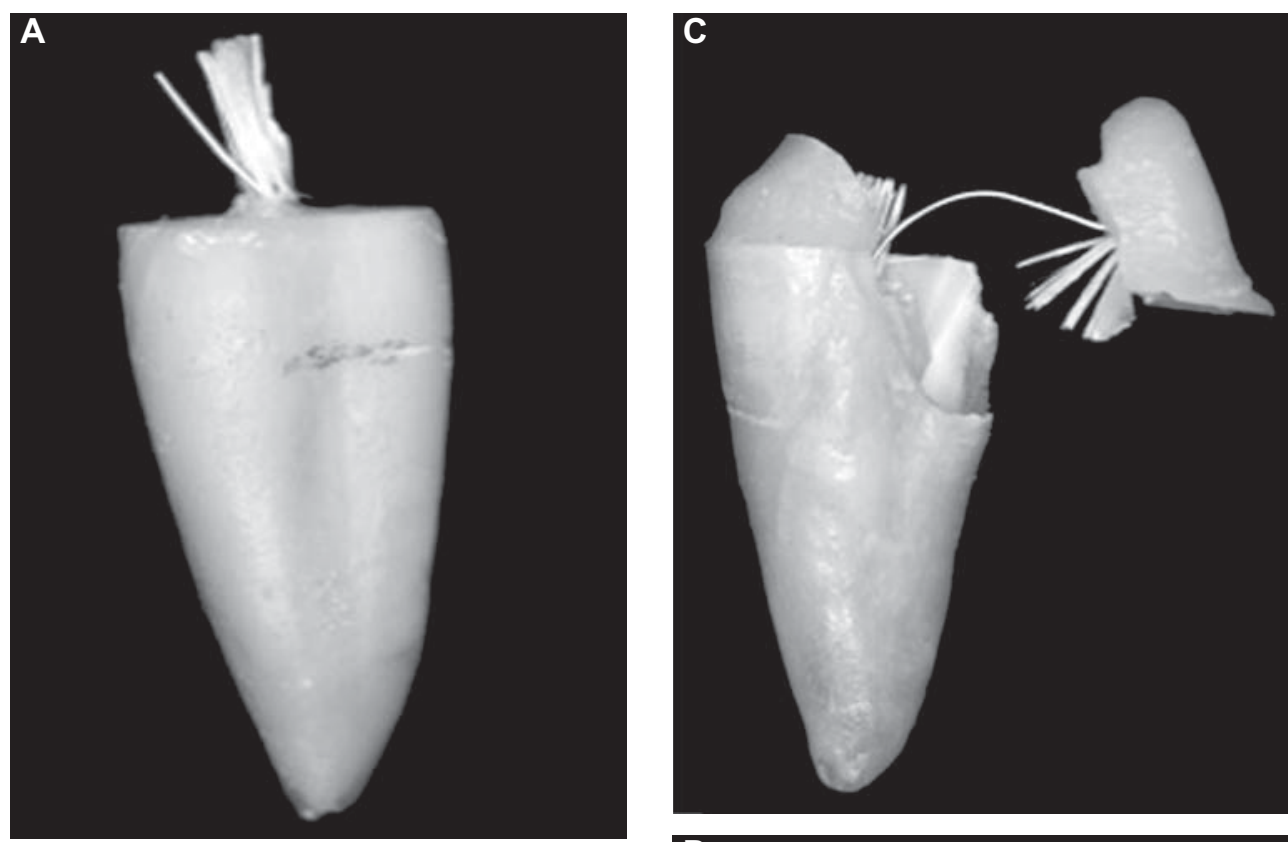

B

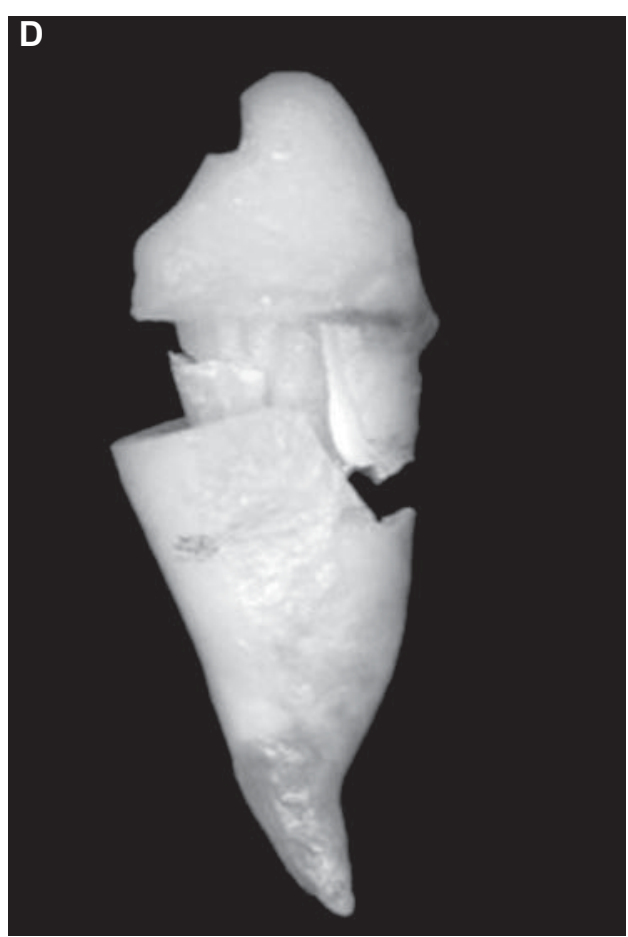

Figure 5- Predominant failure patterns in group $1(A)$, group $2(B)$, group $3(C)$ and group $4(D)$

\section{DISCUSSION}

In an attempt to avoid early loss and reestablish function of the weakened root remnant in the oral cavity, different materials and restorative techniques have been combined $5,16,18,23,26$. Among these materials and techniques, it has been suggested that the use of chemically compatible adhesive restorative components, with mechanical properties closer to those of dentin, would favor preservation of the $\operatorname{root}^{3,13,17,21,29}$.

In this study, the use of the glass fiber post 
associated with adhesive materials by different restorative techniques was assessed. None of the restoration techniques for weakened roots provided root fracture resistance values similar to those found for the non-weakened control group. The restorative techniques used to reconstruct the weakened roots were shown to have no significant effect on the fracture strength and failure mode. Thus, the null hypothesis was rejected. This result is explained because the root resistance to fracture is directly related to the volume of remaining dentin ${ }^{21,30}$. Other studies have shown that no material was capable of recovering root strength when compared with healthy dentin ${ }^{18,23,26,30}$.

It has been demonstrated that when a single tooth is subjected to a load applied at an angulation of $45^{\circ}$ in relation to its long axis, the greatest compression stress and tension occurs at the lingual and vestibular surfaces of the coronal third of the root $^{8}$. In weakened roots this observation could be even more critical, considering the dentinal loss in the coronal third $18,23,26,29,30$. In this study, the results obtained corroborate the findings in the literature consulted.

The failure modes were characterized as repairable (displacement of the nucleus or root fracture beyond the root cervical level) or irreparable (fracture below the cervical third of the root, vertical or oblique fracture and horizontal facture in the middle and apical thirds indication tooth extraction).

In the control group, $100 \%$ of the failures were represented by post and/or core fracture. No root fracture was observed. Whereas in the experimental groups II and IV, root fracture occurred in all specimens and the percentage of repair was $80 \%$ for group II and $50 \%$ for group IV. In group III, half of the specimens presented root fracture and a percentage of $60 \%$ repair was observed. The root fractures in the experimental groups could be explained by their weakening. In all groups, the majority of the failures revealed a higher concentration of stresses on the cervical third of the root.

The group that used composite resin increments and glass fiber post (group II) showed the nearest percentage of reparation of the control group compared with groups III (main glass fiber post with accessories fiber posts) and IV (anatomic post). A possible explanation for this result could be the fact of both groups III and IV were reconstructed with a greater amount of resin cement than group II where only the main glass fiber post was cemented with resin cement. The application of a large volume of cement in the root canal generated high stress at the adhesive interfaces due to the high polymerization shrinkage, and could lead to debonding. Therefore, the adhesive failures may have reduced the stress distribution along the root canal and may have played a decisive role on the failure mode of the flared roots. Similar result was found by Clavijo, et al. ${ }^{5}$ (2009) although the present work used human teeth instead of bovine teeth.

Clinically, the core and post are covered by a crown as final restoration. The presence of the crown is fundamental when there is a dentinal remnant of 1-2 mm, in order to obtain the ferrule effect ${ }^{14,19,21}$. This effect is understood as an action of cervical embracement of the crown on the $360^{\circ}$ of the dentinal root preparation, and it is recommended to improve the integrity of endodontically treated teeth ${ }^{14}$. However, in structurally debilitated endodontically treated teeth, with narrow canals, the ferrule effect did not result in additional benefit ${ }^{10,11,26}$. Similarly, there is no axial cervical dental structure for fabricating an efficient ferrule effect on roots with widened canals. Therefore, in this study, no ferrule effect was conferred and the specimens were loaded directly on the core.

This in vitro study presented the limitations common to tests conducted in human teeth, such as specific dimensions, static compressive load and fixed angulation. Clinical extrapolation of the results must be done judiciously and prudently since it is not possible to simulate all the conditions of the oral environment. The final decision about the use of any restorative technique must take into account the variables related to each patient, such as occlusion, masticatory force, level of alveolar bone insertion and presence of parafunctional habits, in order to increase the longevity of endodontically treated teeth with weakened roots.

\section{CONCLUSIONS}

Based on the mechanical test applied and the results obtained, it may be concluded that:

None of the techniques of weakened root reconstruction with intraradicular posts improved root strength;

The thickness of the dental remainder is the preponderant factor in maintaining resistance to fracture;

Among the techniques evaluated in this study, the incremental technique was suggested as being the most recommendable protocol, since the type of fracture that occurred allowed the dental remnant to be repaired.

\section{REFERENCES}

1- Amaral M, Santini MF, Wandscher V, Zogheib LV, Valandro LF. Effect of coronal macroretentions and diameter of a glass-FRC on fracture resistance of bovine teeth restored with fiber posts. Minerva Stomatol. 2009;58:99-106.

2- Asmussen E, Peutzfeldt, Heitmann T. Stiffness, elastic limit, and strength of newer types of endodontic posts. J Dent. 1999;27:275-8. 
3- Cecchin D, Farina AP, Guerreiro CA, Carlini-Júnior B. Fracture resistance of roots prosthetically restored with intra-radicular posts of different lengths. J Oral Rehabil. 2010;37:116-22.

4- Chalifoux PR. Esthetic restoration of endodontically treated teeth: factors that affect prognosis. J Esthet Dent. 1998;10:75-83. 5- Clavijo VG, Reis JM, Kabbach W, Silva AL, Oliveira Junior OB, Andrade MF. Fracture strength of flared bovine roots restored with different intraradicular posts. J Appl Oral Sci. 2009;17:574-8.

6- Grandini S, Sapio S, Simonetti M. Use of anatomic post and core for reconstructing an endodontically treated tooth: a case report. J Adhes Dent. 2003;5:243-7.

7- Gutmann JL. The dentin-root complex: anatomic and biologic considerations in restoring endodontically treated teeth. J Prosthet Dent. 1992;67:458-67.

8- Holmes DC, Diaz-Arnold AM, Leary JM. Influence of post dimension on stress distribution in dentin. J Prosthet Dent. 1996;75:140-7.

9- Hornbrook DS, Hastings JH. Use of bondable reinforcement fiber for post and core build-up in an endodontically treated tooth: maximizing strength and aesthetics. Pract Periodontics Aesthet Dent. 1995; 7:33-42.

10- Katebzadeh N, Dalton BC, Trope M. Strengthening immature teeth during and after apexification. J Endod. 1998;24:256-9.

11- Kimmel SS. Restoration of endodontically treated tooth containing wide or flared canal. NY State Dent J. 2000;66:36-40. 12- King PA, Setchell DJ. An in vitro evaluation of a prototype CFRC prefabricated post developed for the restoration of pulpless teeth. J Oral Rehabil. 1990;17:599-609.

13- Lassila LV, Tanner J, Le Bell AM, Narva K, Vallittu PK. Flexural properties of fiber reinforced root canal posts. Dent Mater. 2004;20:29-36.

14- Libman WJ, Nicholls JI. Load fatigue of teeth restored with cast posts and cores and complete crowns. Int J Prosthodont. 1995;8:155-61.

15- Lui JL. Composite resin reinforcement of flared canals using light-transmitting plastic posts. Quintessence Int. 1994;25:313-9. 16- Maccari PC, Cosme DC, Oshima HM, Burnett LH Jr, Shinkai RS. Fracture strength of endodontically treated teeth with flared root canals and restored with different post systems. J Esthet Restor Dent. 2007;19:30-6.

17- Malferrari S, Monaco C, Scotti R. Clinical evaluation of teeth restored with quartz fiber-reinforced epoxy resin posts. Int J Prosthodont. 2003;16:39-44.

18- Marchi GM, Paulillo LA, Pimenta LA, De Lima FA. Effect of different filling materials in combination with intraradicular posts on the resistance to fracture of weakened roots. J Oral Rehabil. $2003 ; 30: 623-9$
19- Martinez-Insua A, Silva L, Rilo B, Santana U. Comparison of the fracture resistances of pulpless teeth restored with a cast post and core or carbon-fiber post with a composite core. J Prosthet Dent. 1998; 80:527-32.

20- Mendoza DB, Eakle WS, Kahl EA, Ho R. Root reinforcement with a resin-bonded preformed post. J Prosthet Dent. 1997;78:10-4. 21- Milot $P$, Stein RS. Root fracture in endodontically treated teeth related to post selection and crown design. J Prosthet Dent. 1992;68:428-35.

22- Monticelli F, Grandini S, Goracci C, Ferrari M. Clinical behavior of translucent-fiber posts: a 2-year prospective study. Int J Prosthodont. 2003;16:593-6.

23- Newman MP, Yaman P, Dennison J, Rafter M, Billy E. Fracture resistance of endodontically treated teeth restored with composite posts. J Prosthet Dent. 2003;89:360-7.

24- Oliveira JA, Pereira JR, Valle AL, Zogheib LV. Fracture resistance of endodontically treated teeth with different heights of crown ferrule restored with prefabricated carbon fiber post and composite resin core by intermittent loading. Oral Surg Oral Med Oral Pathol Oral Radiol Endod. 2008;106:52-7.

25- Rosentritt M, Fürer C, Behr M, Lang R, Handel G. Comparison of in vitro fracture strength of metallic and tooth-coloured posts and cores. J Oral Rehabil. 2000;27:595-601.

26- Saupe WA, Gluskin AH, Radke RA Jr. A comparative study of fracture resistance between morphologic dowel and cores and a resin-reinforced dowel system in the intraradicular restoration of structurally compromised roots. Quintessence Int. 1996;27:48391.

27- Sirimai S, Riis DN, Morgano SM. An in vitro study of the fracture resistance and the incidence of vertical root fracture of pulpless teeth restored with six post-and-core systems. J Prosthet Dent. 1999;81:262-9.

28- Solomon CS, Osman YI. Aesthetic restoration of the compromised root: a case report. SADJ. 2003;58(10):370, 373$6,381$.

29- Yoldas O, Akova T, Uysal H. An experimental analysis of stresses in simulated flared root canals subjected to various postcore applications. J Oral Rehabil. 2005;32:427-32.

30- Zogheib LV, Pereira JR, Valle AL, Oliveira JA, Pegoraro LF. Fracture resistance of weakened roots restored with composite resin and glass fiber post. Braz Dent J. 2008;19:329-33. 